$$
\operatorname{con}-9207155--2
$$

\title{
AN ACOUSTO-ULTRASONIC NDE TECHNIQUE FOR MONITORING MATERIAL ANISOTROPY*
}

\author{
H.-T. Chien, S.-H. Sheen, and A. C. Raptis
}

ANL/CP- -75819

DE93 002994

Materials and Components Technology Division ARGONNE NATIONAL LABORATORY

Argonne, Illinois 60439
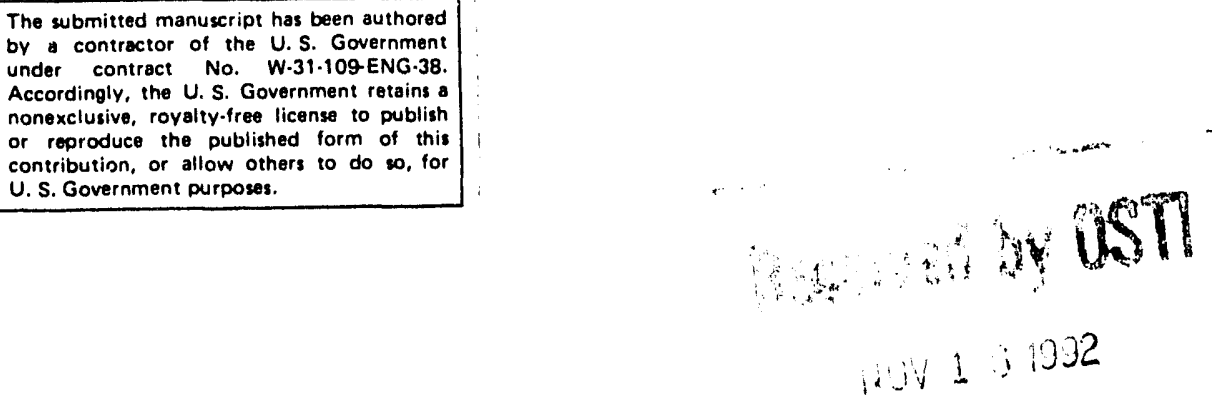

August 1992

To be presented at the 19th Annual Review of Progress in Quantitative Nondestructive Evaluation, sponsored by Iowa State University and Ames Laboratory, members of the Institute for Physical Research and Technology at Iowa State University, in cooperation with the Air Force Wright Research and Development Center/Material Laboratory, ANST, DOE, FAA, NIST, NSF, and others.

*This work was supported by the U.S. Department of Energy, Office of Fossil Energy, AR\&TD Material Program, and Assistant Secretary for Conservation and Renewal Energy, Office of Transportation Systems, as part of the Ceramics Technology for Advanced Heat Engined project of Advanced Materials Development Program, under Contract W-31-109-Eng-38.

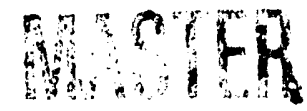




\title{
AN ACOUSTO-ULTRASONIC NDE TECHNIQUE FOR MONITORING MATERIAL ANISOTROPY
}

\author{
Hual-Te Chien, Shuh-Haw Sheen, and Apostolos C. Raptis \\ Materials and Components Technology \\ Argonne National Laboratory \\ Argonne, IL 60439
}

\section{INTRODUCTION}

Due to the advantages of higher strength-weight ratio, larger stiffness, stronger corrosion and wear resistance, longer fatigue life, and better thermal insulation, fiber-reinforced composite materials have been widely applied, for example, in the industries of airplane, automobile, marinecraft, spacecraft, and construction. How to evaluate material properties, to detect defects, and to predict material life of usage are a important but tedious work to do. In the last decade considerable attention has been focused on using acousto-ultrasonic ( $\mathrm{AU}$ ) and leaky-Lamb wave techniques to evaluate material properties or changes in material properties due to fiber misorientation, flaws and defects, fiber/matrix debonding, or external loading $[1,2,3,4]$. A general summary of the AU approach, with bibliography, was presented by Vary [5]. A typical experimental setup with a personal computer (PC) to analyze the AU waveforms was described by Kiernan and Duke [6]. The propagation of Leaky-Lamb waves in fiber-reinforced composites was investigated theoretically and experimentally by Chimenti and Nayfeh $[7,8]$. A general discussion of elastic waves in solids can be found in Ref. [9].

To date, most studies have examined the available energy of the signal waveform, a combined waveform of bulk, extension, and Lamb waves, by measuring the stress wave factor (SWF), which requires sophisticated instrumentation and signal processing $[1,5,6]$. In this study an alternative approach for $A U$ nondestructive evaluation (NDE) technique, which requires minimal instrumentation and analysis, 
has been developed to monitor the material anisotropy of fiber-reinforced composites. Instead of measuring the SWF, the $\mathrm{AU}$-wave time of flight (TOF) is measured and related to material properties and fiber orientation. Two longitudinal transducers (0.5 MHz) are placed on the same surface of composite to excite and catch Lamb waves. Exciting under the first critical frequency of the Lamb wave for the material, two fundamental modes are excited. The slower one is the first antisymmetric mode and the faster one is the first symmetric mode, which is nearly nondispersive and has a phase velocity very close to that of the bulk longitudinal wave. As the transducer separation increases, the measured time-domain AU signal of these two modes separates more distinctly.

The experiments were conducted with Kevlar $49^{1}$, styles 120 and 181 . The distance effect was also studied and shows that the TOF dependence on transducer separation is linear. Thus, phase velocities, which depend strongly on fiber orientation with respect to wave propagation direction, in the media can be accurately determined from either the direct measurements of the TOF or the slopes of the distance-effect curves. If the wave propagates away from the fiber direction, a slower but more attenuated wave is observed. Theoretical solutions of bulk longitudinal wave are calculated by solving an eigenproblem with known material properties. Good agreement is obtained between experimental and theoretical results and shows that this technique is a better approach to determining material anisotropy than the SWF method does. Because that TOF is sensitive to the fiber orientation and the fiber/matrix interface, this AU-NDE technique has a great potential in detecting defects and debonding of fiber-reinforced composite materials.

\section{THEORETICAL STUDY}

Due to the anisotropic nature of composite materials, analysis of wave propagation in them is relatively complicated. The three-dimensional Hook's law must be considered in generating the three-dimensional wave equation. From the principle of conservation of energy for a homogeneous, anisotropic medium, acoustic fields in a solid are fully described by stress equations of motion, or so-called Christoffel equations, of a continuous medium with the absence of body forces [9],

$$
\frac{\partial \sigma_{i j}}{\partial x_{j}}=\rho \frac{\partial^{2} u_{i}}{\partial t^{2}}
$$

and the associated linear constitutive relations are

$$
\sigma_{i j}=C_{i j k \ell} \epsilon_{k \ell}
$$

\footnotetext{
${ }^{1}$ Kevlar is a registered trademark of E. I. Du Pont de Nemours \& Co.
} 


$$
\epsilon_{k \ell}=\frac{1}{2}\left(\frac{\partial u_{k}}{\partial x_{\ell}}+\frac{\partial u_{\ell}}{\partial x_{k}}\right),
$$

where $\sigma_{i j}$ represents the stress, $C_{i j k \ell}$ the elastic stiffness constant, $\epsilon_{i j}$ the strain, $u_{i}$ the displacement, $i, j, k=1,2,3, \rho$ the material density, and $x_{i}=\left(x_{1}, x_{2}, x_{3}\right)$ the coordinate system.

If uniform plane waves are considered to propagate in a lossless unbounded (infinite) medium with the same wave numbers in all directions, the field is as follows:

$$
u_{j}=U_{j} \exp ^{i\left(\omega t-\xi I \cdot x_{i}\right)}
$$

where $I=\left(l_{1} x_{1}, l_{2} x_{2}, l_{3} x_{3}\right)$ represents the unit vector of propagation direction, $\xi$ the wave number, $\omega$ the circular frequency $(=2 \pi f)$, and $i=\sqrt{-1}$. Substituting Eq. (3) into Eq. (1) yields three linear homogeneous coupled equations

$$
\left(\Gamma_{i j}-\rho c^{2} \delta_{i j}\right) u_{j}=0
$$

where

$$
\Gamma_{i j}=l_{i p} C_{p q} l_{q j}
$$

and $c=\omega / \xi$ is the phase velocity. $l_{i p}$ is the gradient operator matrix and is given as

$$
l_{p i}=\left[\begin{array}{cccccc}
l_{1} & 0 & 0 & 0 & l_{3} & l_{2} \\
0 & l_{2} & 0 & l_{1} & 0 & l_{3} \\
0 & 0 & l_{3} & l_{2} & l_{1} & 0
\end{array}\right]
$$

with $l_{p i}=l_{i p}^{\top}$. The abbreviated subscript notations, or the so-called Vogit convention, are used to simplify the transformation of material constants and the matrix manipulations. These notations relate material constants $C_{p q}, p, q=1, \ldots, 6$, to $C_{i j k \ell}$ with $1 \rightarrow 11,2 \rightarrow 22,3 \rightarrow 33,4 \rightarrow 23,5 \rightarrow 13$, and $6 \rightarrow 12$, respectively.

By solving the eigenproblem in Eq. (4), the bulk-wave velocities, associated with a longitudinal wave ( $P$-wave) and two shear waves ( $S H$ and $S V$-waves), can be obtained. If the medium has a finite thickness $d$ and plane waves propagating along the $x_{1}$-direction are independent of $x_{2}$, the phase velocities of Lamb waves can be obtained by solving Eq. (4) and simultaneously satisfying the asspciated boundary conditions, i.e., free surface traction on both surfaces of the media $[7,8]$. The phase velocities of Lamb waves are dispersive to the frequency and also the azimuthal angle. However, by limiting the frequency below the first critical frequency, there are only two fundamental Lamb modes can be detected: one is the first antisymmetric mode with lower phase velocity, which is dispersive but is not sensitive to the changing of azimuthal angle; the another is the first symmetric mode with higher speed, which is 
nearly nondispersive and very close to that of the associated bulk longitudinal wave, and is also sensitive to the changing of azimuthal angle. For this reason, in this study, we only compare the experimental measurements with the theoretical results of bulk longitudinal wave.

\section{EXPERIMENTAL PROCEDURE}

Because only TOF is measured in this experiment, the experimental setup and signal processing are much simpler than these needed for the SWF method. In this experiment, a Wavetek pulse generator is used to excite a longitudinal-wave, or $\mathrm{P}$-wave, transducer with a pulse width of $1.2 \mu \mathrm{sec}$. Two transducers, one acting as a transmitter and the another as a receiver, are attached normal to the surface on the same side of the composite with a well-defined separation distance between the transmitter and receiver (DTR). Both transducers have a center frequency of $0.5 \mathrm{MHz}$ with 0.5 inch in length and 1.0 inch in width. To obtain a good signal within a reasonably accessible space, the separation distance is chosen as 3 inches. The received signal is magnified by a differential $\mathrm{AC}$ amplifier before being sampled and displaced on a LeCroy 9400 digital oscilloscope. The complete experimental setup is illustrated in Fig. 1.

Due to the highly attenuated nature and material anisotropy of composites, the surface smoothness, couplant condition and pressure of the transducer attachment must be examined and established before the experiment. In most cases surface finishing was not necessary because the composite surface was adequately smooth due to surface coating. Ultragel couplant was selected to attach the transducers. To maintain consistency in the TOF readings, a constant pressure was loaded on both transducers. A distance-measurement fixture was used to house the transducers at selected separations.

The line joining the transmitter and the receiver is defined as the wave propagation direction. In Fig. 2 , the $x_{1}$-axis defines the direction that is parallel to the $0^{\circ}$ fiber orientation of the specimen, and the angle between the propagation direction and the $x_{1}$-axis is called the azimuthal angle, $\Theta$. Figure 2 also shows the fiber orientation and setup of composite. A $360^{\circ}$ protractor is fixed on the transducer fixture to keep track of the azimuthal angle. The linear readings of the TOF were taken from the average of 500 samplings. Phase velocities, then, were obtained by collecting TOF at different azimuthal angles to show material anisotropy. Scans on both sides of the composite were made to verify experimental consistency and it was 
found that the data obtained from one side of the composite was accurate enough to identify the material anisotropy. The distance effect was also studied by measuring the TOF with variations of DTR for a fixed azimuthal angle. The slopes of the TOF curve were then related to the phase velocities of the longitudinal bulk wave.

\section{RESULTS AND DISCUSSION}

Experiments were conducted for Kevlar 49 (styles 120 and 181), composites to demonstrate this AU technique. Kevlar 49, style 120, is a composite having 28 plies in the warp direction with plain weave in Fiberite MXM7714 resin, while style 181 has 11 plies in the warp direction with 8-harness satin weave in Cucom 919 resin. Both of them are bidirectional composites and their material properties are:

$$
\begin{gathered}
C_{11}=C_{22}=40.09 \mathrm{GPa}, \quad C_{33}=5.54 \mathrm{GPa} \\
C_{12}=1.88 \mathrm{GPa}, \quad C_{13}=C_{23}=1.68 \mathrm{GPa} \\
C_{44}=C_{55}=2.1 \mathrm{GPa}, \quad C_{66}=2.3 \mathrm{GPa}, \quad \rho=1.6 \mathrm{~g} / \mathrm{cm}^{3} .
\end{gathered}
$$

Figure 3 shows the AU waveforms for Kevlar 49, style 120, measured with DTRs of 3 and 8 inches. A clear separation of these two groups is observed when DTR is 8 inches. The distance-dependent TOFs for both Kevlar styles are given in Fig. 4, and the results show that the dependence of TOF on transducer separation is linear. Therefore, from the inverse of the slope of the linear dependences, the phase velocities of longitudinal bulk wave for various azimuthal angles are determined. However, because the transducers are 0.5 inches in length, the TOF for DTR $\leq 1.5$ inches is difficult to resolve. Furthermore, the linear line does not cross the origin, chiefly because the process is no longer of the AU mode but is under a pulse-echo mode. The slope for wave propagation along $\Theta=0^{\circ}$ is smaller than that along $45^{\circ}$; thus, the phase velocity in $0^{\circ}$ is faster than that in $45^{\circ}$. This can also be verified by examining their TOF of AU waveform.

Under the first critical frequency, two Lamb modes were generated: one is the first antisymmetric mode with a slower velocity; the another is the first symmetric mode with a faster speed. If the ultrasonic waves propagate away from the fiber direction, these two modes still can be observed but travel at a slower velocity and display a higher attenuation. Figure 5 shows the waveforms for $\Theta=0^{\circ}, 45^{\circ}$, and $90^{\circ}$ for a Kevlar 49, style 120, composite with a DTR of 3 inches. Because the composite has a bidirectional symmetry, the waveforms for $\Theta=0^{\circ}$ and $90^{\circ}$ are similar and have TOFs of 14.92 and $14.83 \mu \mathrm{sec}$, respectively. If $\Theta=45^{\circ}$, the signal suffers a higher attenuation and also shows a slower TOF of $18.14 \mu \mathrm{sec}$. Therefore, the TOF of an AU 


\begin{tabular}{||c|c|c|c|c||}
\hline \hline \multirow{2}{*}{$\begin{array}{c}\text { Approach } \\
\text { Method }\end{array}$} & \multicolumn{2}{|c|}{ Kevlar 49 120 } & \multicolumn{2}{|c|}{ Kevlar 49 181 } \\
\cline { 2 - 5 } & $0^{\circ}$ & $45^{\circ}$ & $0^{\circ}$ & $45^{\circ}$ \\
\hline TOF & 4.882 & 3.892 & 4.806 & 3.762 \\
\hline Slope & 4.908 & 3.666 & 4.798 & 3.579 \\
\hline Theory & 5.006 & 3.815 & 5.006 & 3.815 \\
\hline
\end{tabular}

Table 1: Phase velocities of Kevlar 49, style 120 and 181, composites from TOF and slope measurements, and theoretical approaches, respectively (Unit: Km/sec).

waveform is dispersive with respect to the azimuthal angle. Because the TOF and attenuation of $\mathrm{AU}$ signals may be related to the fiber direction and bonding condition, it is possible to use this AU-NDE technique to study the fiber/matrix interface, the detection of debonding and defects, and the effects due to external loading.

Such phase-velocity verses azimuthal-angle curves for both sides of the Kevlar 49 (style 120) composite panel are given in Fig. 6, along with the theoretical results obtained by solving the eigenfunction in Eq. (4) if the material properties are defined. Figure 7 gives the phase-velocity curves and theoretical results for the Kevlar 49 (style 181) composite panel. Good agreement between theoretical results and experimental data is obtained, and the phase velocity measurements based on the phase-velocity curves from both sides of a composite and on the slope of linear curves from the distance effect show high accuracy and consistency. Table 1 shows summary of the phase velocities obtained from these three approaches for the Kevlar 49 composite materials measured at $\Theta=0^{\circ}$ and $45^{\circ}$. The TOF approach is easier for obtaining the phase velocity and dispersion curve, while slope approach requires the measurements at different locations but gives better results. Differences between the experimental and theoretical results may be caused by the accuracy of the material constants and of the TOF measurements. A more sophisticated method for measuring TOF has to be used, however, agreement is still good.

\section{CONCLUSIONS}

A simpler and better way of monitoring the anisotropy of fiber-reinforced composite materials, based on the acousto-ultrasonic approach, is presented. In this approach, time of flight of the acousto-ultrasonic waves, rather than the stress wave factor, is measured. Two fundamental Lamb modes are generated under the first critical frequency: one is the first antisymmetric mode traveling with a slower velocity while the another is the first symmetric mode traveling with a faster speed. The later one is sensitive to the azimuthal angle and nearly nondispersive, and has a phase 
velocity very close to that of the bulk longitudinal wave of the material. Experimental data measured from two methods, TOF measurement and slope method, are compared with the theoretical results; a good agreement is obtained for monitoring the material anisotropy. There is a great potential for this AU approach in material-property evaluation and in quantitative measurements of defects and debonding of fiber-reinforced composites. However, more studies are needed to better understand the effect of the fiber/matrix bonding on the measurements and to extract more information from the AU signals.

\section{ACKNOWLEDGMENT}

Work sponsored by USDOE/Fossil Energy/Advanced Research Technology Development/Materials Program, under Contract W-31-109-Eng-38.

\section{REFERENCES}

[1] J. C. Duke, Jr., editor, Acousto-Ultrasonics - Theory and Application (Plenum Press, New York, 1988).

[2] A. Vary, Mat'l Eva. $\underline{49}, 581$ (1991).

[3] V. K. Choo, S.-H. Sheen, W. P. Lawrence, and H.-T. Chien, in Proceedings of the First Canadian International Composites Conference and Exhibition (Montreal, Canada 1991).

[4] V. K. Choo, S.-H. Sheen, W. P. Lawrence, and H.-T. Chien, in SAMPE Technical em Seminar (Fort Worth/Dallas, USA, 1991).

[5] A. Vary, in Non-destructive Testing of Fiber-Reinforced Plastics Composites, edited by John Summerscales (Elsevier Applied Science, UK), p. 1 (1991).

[6] M. T. Kiernan and J. C. Duke, Jr., Mat'l Eva. 뜨, 1344 (1988).

[7] D. E. Chimenti and A. H. Nayfeh, J. Appl. Mech., Trans. ASME $\underline{33}, 4531$ (1985).

[8] A. H. Nayfeh and D. E. Chimenti, J. Acoust. Soc. Am. 83, 1736 (1988).

[9] B. A. Auld, Acoustic Fields and Waves in Solids, Vol. I\&II (John Wiley, New York, 1973). 


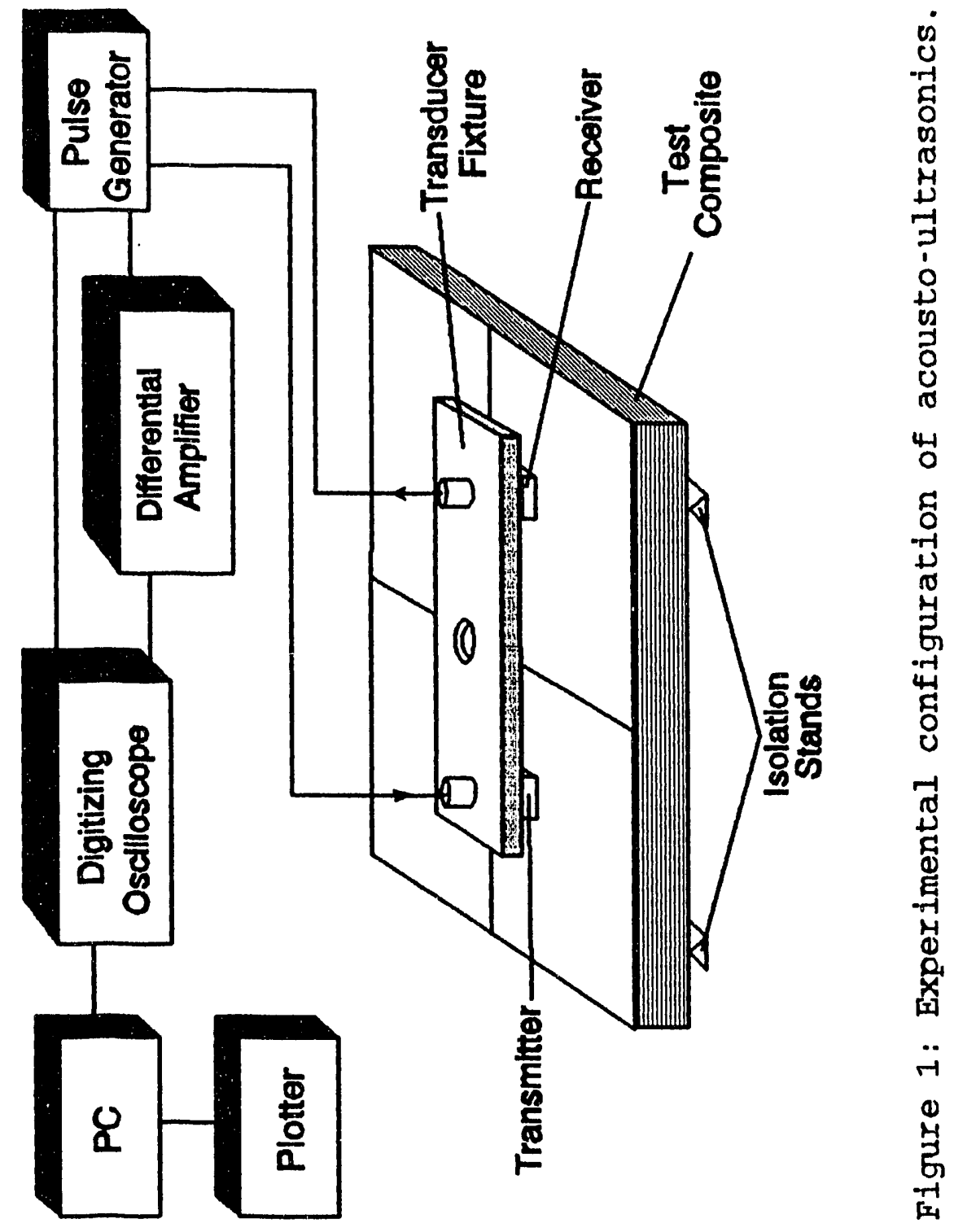




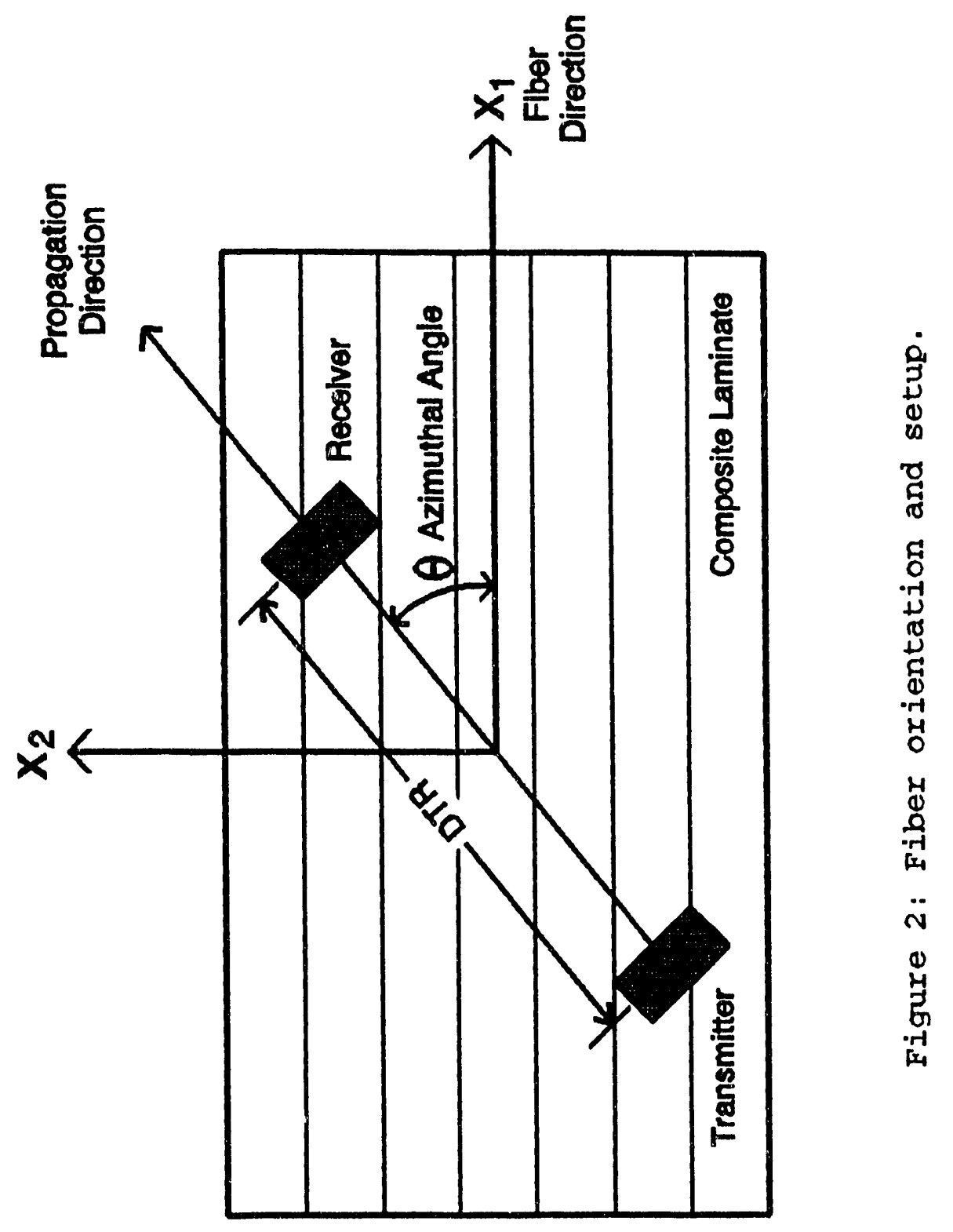



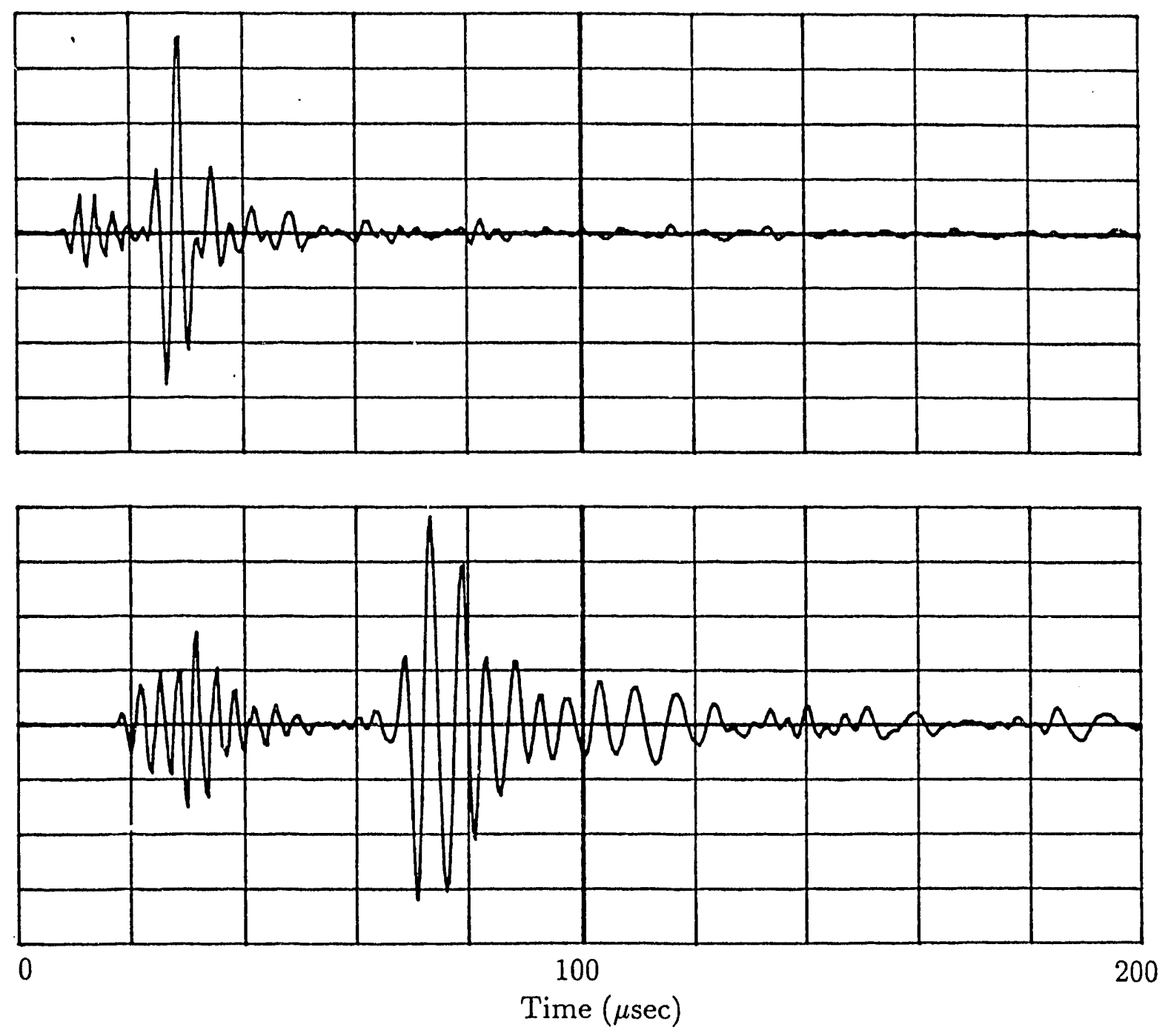

Figure 3: AU waveforms for Kevlar 49, style 120, composite panel with DTR of 3 and $8 \mathrm{in}$. 


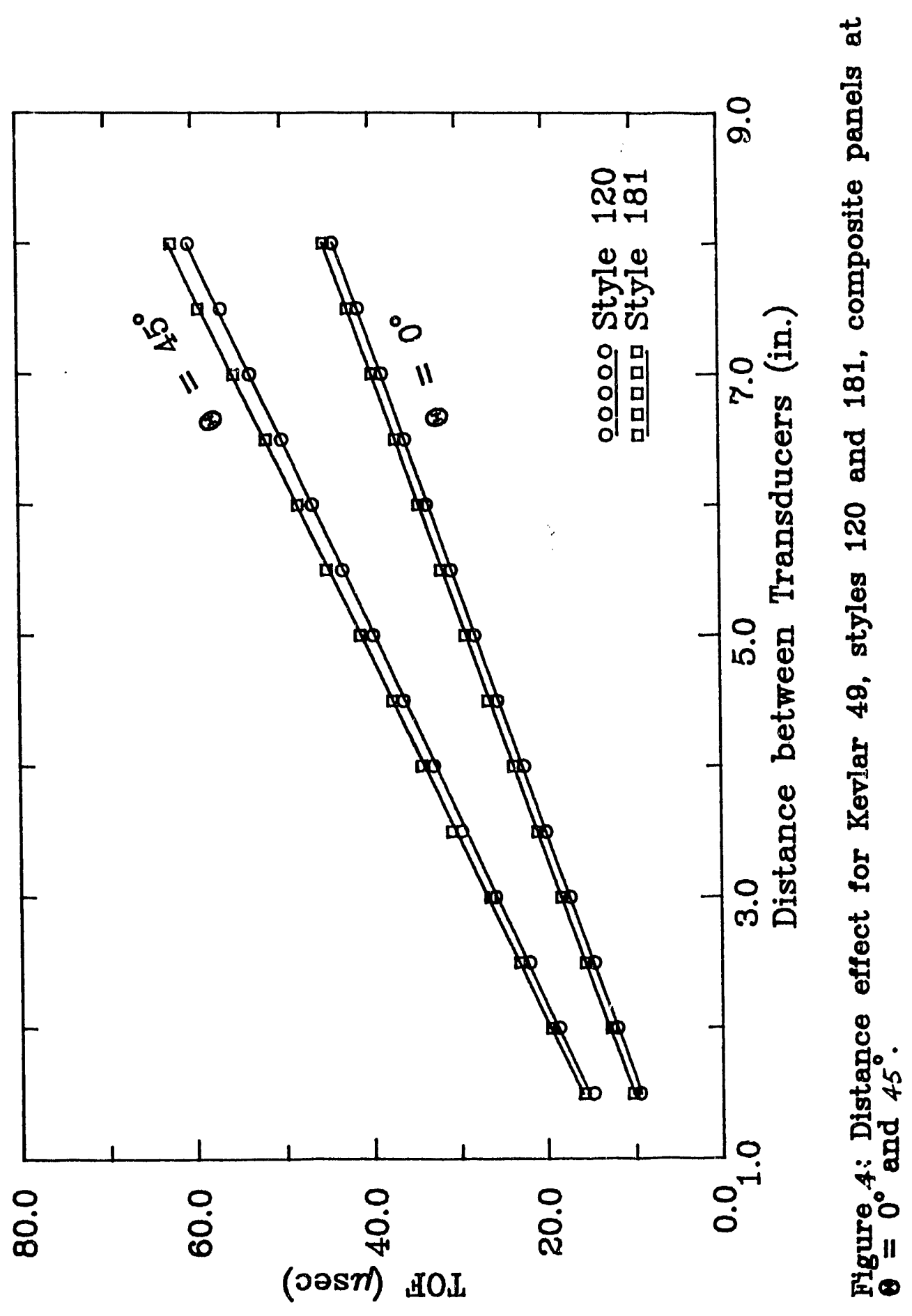



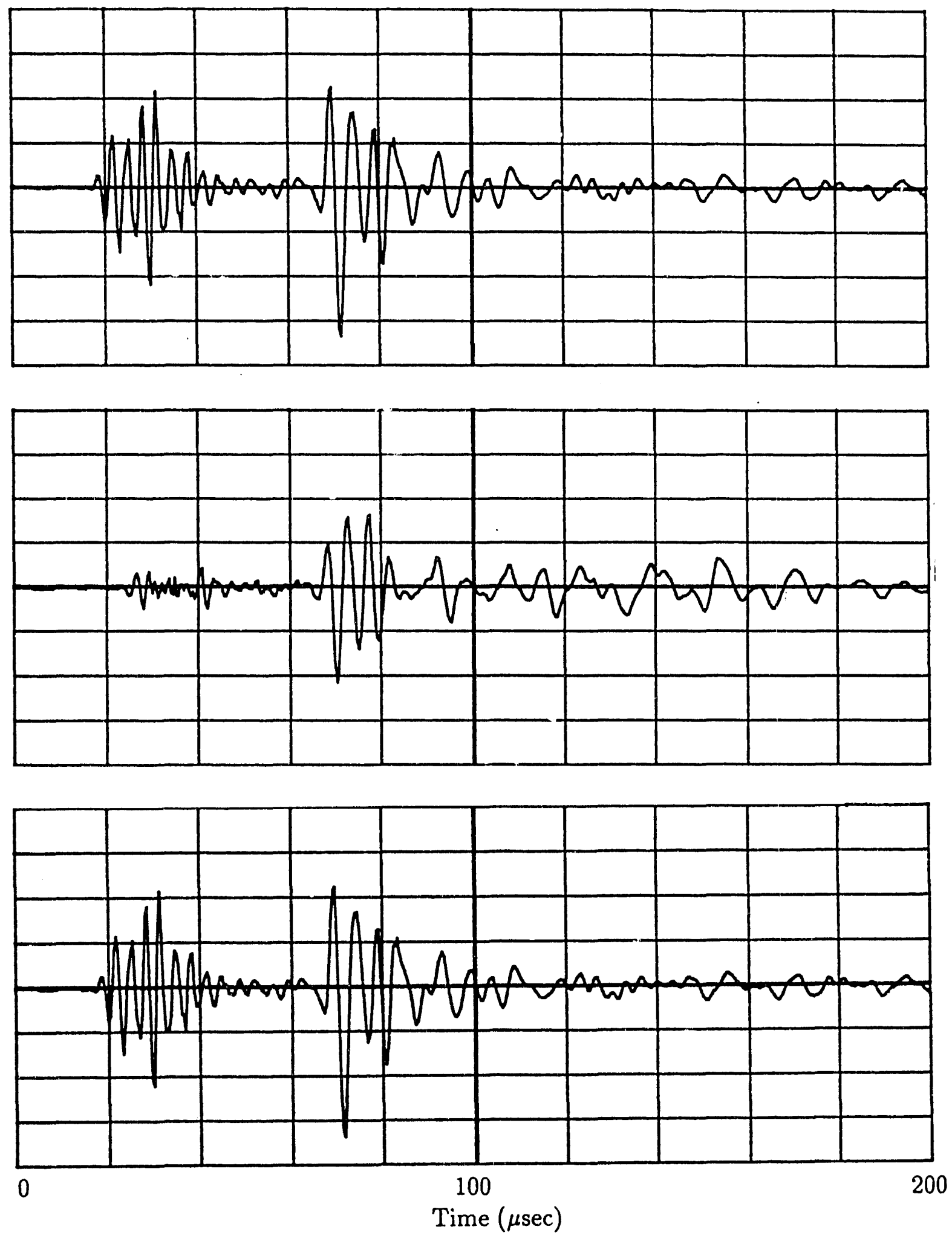

Figure 5: AU waveforms for Kevlar 49, style 120, composite panel at $\Theta=0^{\circ}, 45^{\circ}$ and $90^{\circ}$. 


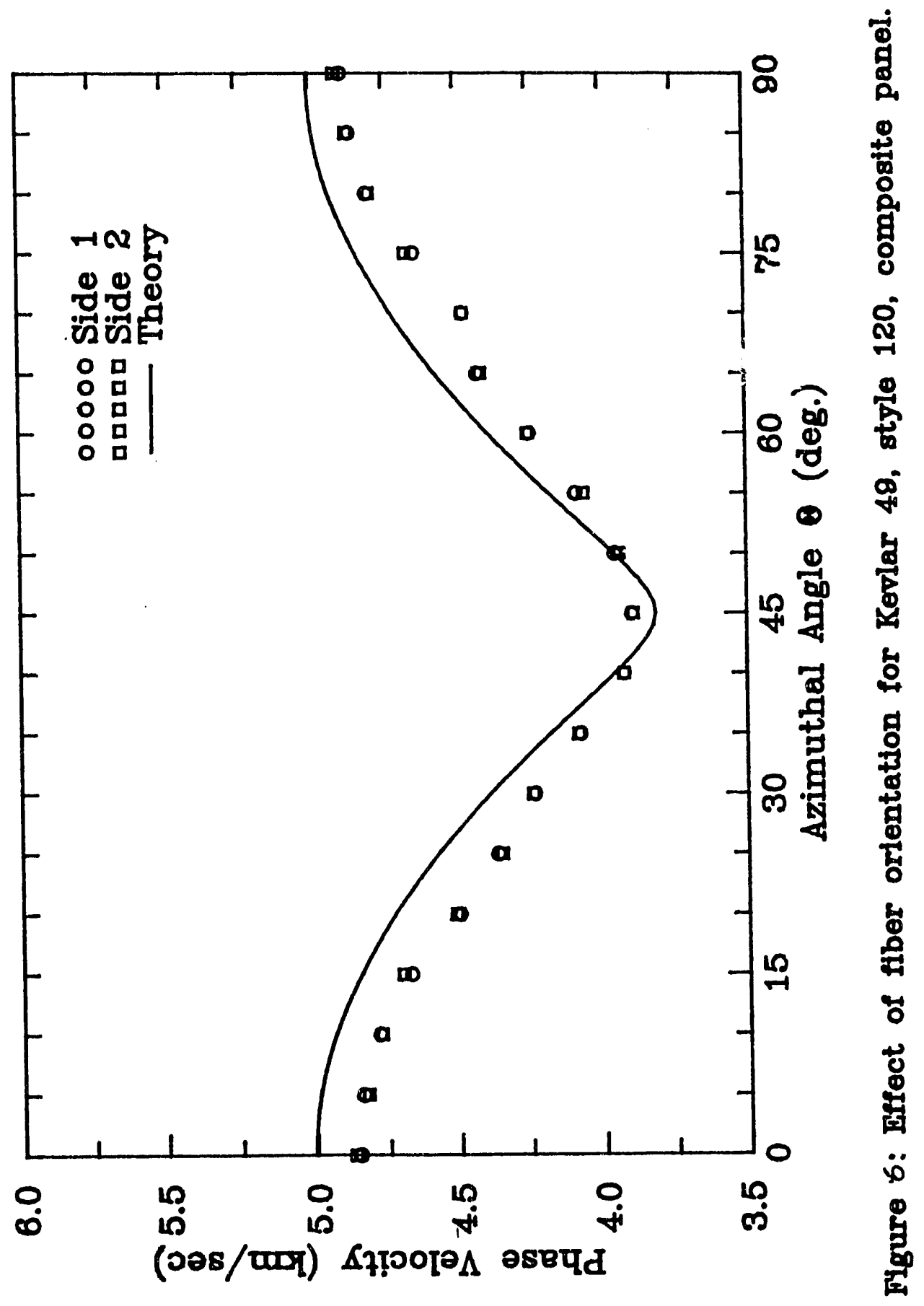




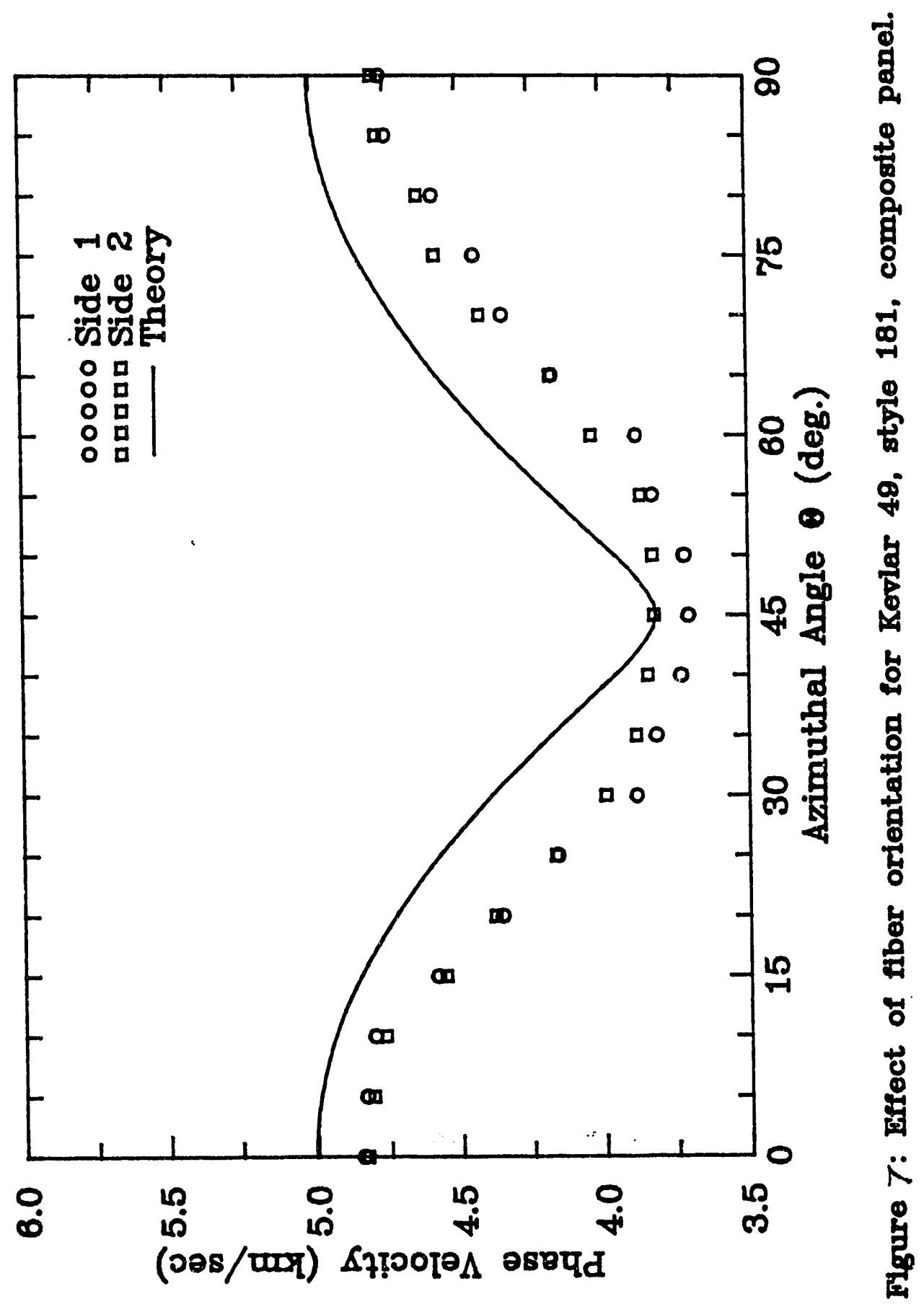



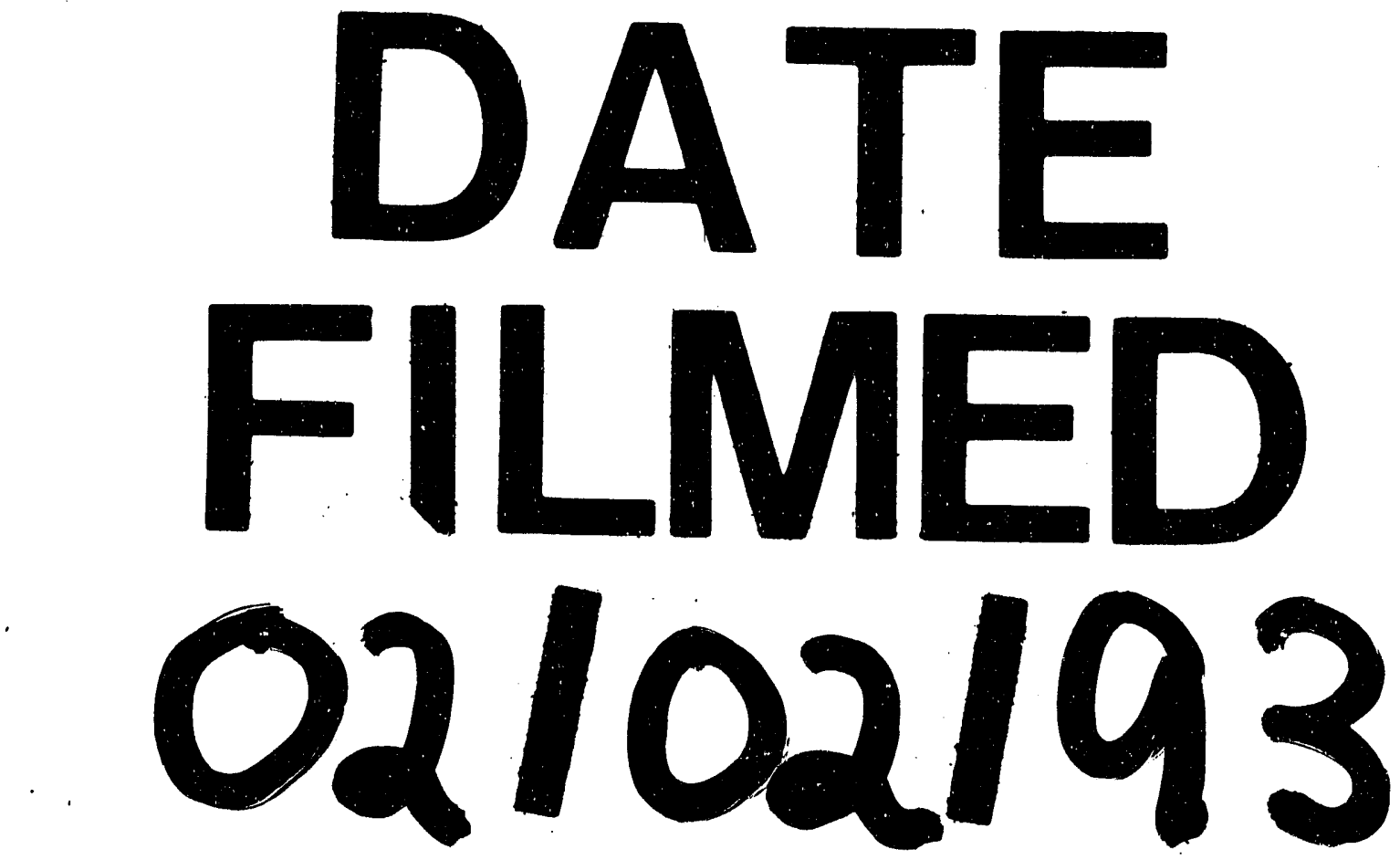
